\title{
Association of leptin receptor gene polymorphisms with post-transplant diabetes mellitus: Short report and literature review
}

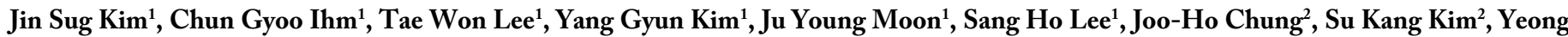 \\ Hoon Kim ${ }^{3}$ and Kyung Hwan Jeong ${ }^{1 *}$ \\ ${ }^{1}$ Division of Nephrology, School of Medicine, Kyung Hee University, Seoul, Korea \\ ${ }^{2}$ Kohwang Medical Research Institute, School of Medicine, Kyung Hee University, Seoul, Korea \\ ${ }^{3}$ Division of Nephrology, School of Medicine, Inje University, Busan, Korea
}

\begin{abstract}
Background: Post-transplant diabetes mellitus (PTDM) is a common and important metabolic complication after renal transplantation. Although genetic variants of the leptin $(L E P)$ and leptin receptor $(L E P R)$ gene have been reported to be associated with insulin resistance and diabetes mellitus, few studies have examined these variants in patients with post-transplant diabetes mellitus (PTDM). In this study, we investigated the association between $L E P$ and $L E P R$ polymorphisms and PTDM in renal transplant recipients. We also reviewed the literature on the genetic variants associated with development of PTDM.

Methods: A total of 301 patients who received renal transplants and had no history of diabetes were included in this study. We analyzed the associations between development of PTDM and the five single nucleotide polymorphisms (SNPs) in LEP(rs1322837 and rs2167270) and LEPR (rs8179183, rs1137100, and rs1137101).

Results: PTDM developed in 48 of the 301 patients studied (15.9\%). Patients with PTDM had significantly higher allele frequency of the LEPR rs1137100*G allele and rs $1137101^{*} \mathrm{G}$ allele. After adjustment for age, gender, and tacrolimus usage, rs113700 and rs 1137101 in $L E P R$ showed significant association with the development of PTDM.
\end{abstract}

Conclusions: $L E P R$ polymorphisms were significantly associated with PTDM in renal transplant recipients. These data suggest that SNPs of $L E P R$ may be associated with the pathogenesis of PTDM and may act as genetic markers for the development of PTDM.

\section{Introduction}

The development of post-transplant diabetes mellitus (PTDM) is a devastating metabolic complication after renal transplantation [1]. It affects $2-50 \%$ of renal transplant recipients and is associated with graft failure, cardiovascular complications, infection, and mortality $[2,3]$. As in type 2 diabetes mellitus (T2DM), decreased insulin secretion, increased insulin resistance, or a combination of both are believed to be involved in PTDM [3,4]. Although various risk factors such as older age, obesity, hepatitis $\mathrm{C}$ infection, and type of immunosuppressive regimen are well established, they do not fully account for the development of PTDM [3]. Recently, many studies have been conducted to analyze genetic polymorphisms as markers for PTDM $[5,6]$. These studies have suggested that the development of PTDM is related to the genotypes of several genes, such as adiponectin $(A D I P O Q)$, transcription factor 7-like 2 (TCF7L2), potassium voltage-gated channel subfamily Q member 1 (KCNQ1), and $\mathrm{C}-\mathrm{C}$ motif ligand 5 (CCL5), which are involved in insulin resistance and sensitivity [7-10]. Leptin (LEP) and leptin receptor $(L E P R)$ gene polymorphisms have been reported to be associated with insulin resistance, obesity, and diabetes mellitus $[11,12]$. However, only a few studies have evaluated the clinical impact of $L E P$ and $L E P R$ polymorphisms on the development of PTDM [13].

In this study, we ascertained whether $L E P$ and $L E P R$ polymorphisms are associated with PTDM in Korean patients who underwent renal transplantation. We also reviewed literature that investigated associations between gene polymorphisms and PTDM in renal transplant recipients.

\section{Methods}

A total of 301 renal transplant recipients were recruited at three transplant centers in the Republic of Korea (Kyung Hee University Medical Center, Kyung Hee University Hospital at Gangdong, and Inje University Busan Paik Hospital) from 2000 to 2009. Patients were excluded when they had a history of diabetes or impaired fasting glucose (fasting glucose level $100-125 \mathrm{mg} / \mathrm{dL}$ ) before transplantation. All study procedures complied with the ethical guidelines of the 1975 Declaration of Helsinki, as revised in 2000 . This study was approved by the ethics review committees of all three transplant centers and written informed consent was obtained from each subject.

Correspondence to: Kyung Hwan Jeong, Division of Nephrology, Department of Internal Medicine, School of Medicine, Kyung Hee University, \#1 Hoeki-Dong, Dongdaemoon-Gu, Seoul, 130-702, Republic of Korea, Tel: +82-2-958-8200, Fax: +82-2-968-1848; E-mail : khjeong@khu.ac.kr

Key words: leptin, leptin receptor, gene polymorphism, post-transplant diabetes mellitus, renal transplantation

Received: January 15, 2017; Accepted: January 26, 2017; Published: January 30 2017 
PTDM was diagnosed based on American Diabetes Association guidelines [14]. SNPs were selected in $L E P$ and $L E P R$ using the NCBI dsSNP database, version 131 (http:// www.ncbi.nlm.nih.gov/SNP) and the database of the International Hapmap Project (http://www.hapmap. org/index.html). Two LEP (rs1322837 and rs2167270) and three LEPR SNPs (rs8179183, rs1137100, and rs1137101) were ultimately selected and used to genotype the patients. Blood samples were collected from each subject and then stored at $-20^{\circ} \mathrm{C}$. Genomic DNA was isolated from blood samples with a commercially available Qiagen DNA extraction kit (Qiagen, Tokyo,Japan). SNP genotyping was conducted by direct sequencing. Genomic DNA was amplified with specific primers for two LEP and three LEPR SNPs (Table 1). The amplified products were sequenced with an ABI PRISM 3730XL analyzer (PE Applied Biosystems, Foster City, Calif, USA), and sequence data were analyzed with SeqManII software (DNASTAR Inc., Madison, Wisc., USA).

\section{Results}

The overall incidence of PTDM in the study population was $17.9 \%$ (54 of 301 patients). Table 1 shows the baseline characteristics of the study population according to the development of PTDM (PTDM vs. non-PTDM group). The mean follow-up duration for all 301 patients was 87.9 months. Patients in the PTDM group were significantly older than those in the non-PTDM group $(45.17 \pm 9.26 v s .38 .58 \pm 11.20$ years, respectively; $\mathrm{p}<0.001)$. Patients in the PTDM group used tacrolimus more frequently than did those in the non-PTDM group $(\mathrm{p}=0.044)$.

Allele frequencies are shown in Table 2. The PTDM group had a significantly higher allele frequency compared to the non-PTDM group for the rs $1137100^{\star} \mathrm{G}$ allele $(\mathrm{OR}=1.924 ; 95 \% \mathrm{CI}: 1.024-1.192 ; \mathrm{p}=$ $0.025)$ and the rs $1137101^{\star} \mathrm{G}$ allele $(\mathrm{OR}=1.131 ; 95 \% \mathrm{CI}$ : $1.045-1.1225$; $\mathrm{p}=0.019$ ). The effect of genotype on development of PTDM remained significant even when adjusting for age, gender, and tacrolimus usage (Table 3). We nest, tested whether the LEPR haplotype was associated with PTDM. To demonstrate pair-wise linkage disequilibrium (LD), we analyzed three SNPs and found that they were in LD. The D' values between rs8179183 and rs1137100, and between rs8179183 and rs1137101, between rs1137100 and rs1137101 were 0.572, 0.655, and 0.876 , respectively. The $r^{2}$ values between SNPs were also calculated.

Table 1. Clinical characteristics of the study population (PTDM vs. non-PTDM).

\begin{tabular}{|l|c|c|c|}
\hline & PTDM (n=54) & $\begin{array}{c}\text { non-PTDM } \\
(\mathbf{n = 2 4 7})\end{array}$ & $\mathbf{p}$ \\
\hline Follow-up duration (months) & $91.54 \pm 85.36$ & $76.85 \pm 75.49$ & 0.208 \\
\hline Age (years) & $45.17 \pm 9.26$ & $38.58 \pm 11.20$ & $<0.001$ \\
\hline Sex (male: female) & $28: 26$ & $155: 92$ & 0.137 \\
\hline BMI (kg/m $\left.{ }^{2}\right)$ & $22.57 \pm 3.39$ & $22.47 \pm 3.47$ & 0.859 \\
\hline Dialysis duration (months) & $33.20 \pm 58.44$ & $23.90 \pm 34.09$ & 0.336 \\
\hline HLA total mismatching (n) & $3.10 \pm 1.54$ & $3.24 \pm 1.53$ & 0.512 \\
\hline HCV (+) (n, \%) & $0(0 \%)$ & $6(2.36 \%)$ & 0.244 \\
\hline Acute rejection (n, \%) & $13(25.49 \%)$ & $44(17.32 \%)$ & 0.197 \\
\hline Calcineurin inhibitor, $\mathrm{n}(\%)$ & $24(45.3 \%)$ & $74(30.8 \%)$ & 0.044 \\
\hline Tacrolimus & $29(53.7 \%)$ & $166(67.2 \%)$ & 0.098 \\
\hline Cyclosporine & & & \\
\hline Antimetabolite, $\mathrm{n}(\%)$ & $10(18.5 \%)$ & $72(29.1 \%)$ & 0.582 \\
\hline Azathioprine & $34(62.9 \%)$ & $165(66.8 \%)$ & 0.702 \\
\hline MMF & & & \\
\hline Serum creatinine (mg/dL) & $1.27 \pm 0.56$ & $1.32 \pm 0.49$ & 0.529 \\
\hline At 6 months after transplantation & $1.29 \pm 0.36$ & $1.37 \pm 0.87$ & 0.507 \\
\hline At 12 months after transplantation & & & \\
\hline
\end{tabular}

PTDM: post-transplantation diabetes mellitus; BMI: body mass index; HLA: human leukocyte antigen; $\mathrm{HCV}$ : hepatitis $\mathrm{C}$ virus; MMF: mycophenolate mofetil
Table 2. Allele frequencies for 5 SNPs in the LEP and LEPR genes in PTDM and nonPTDM subjects.

\begin{tabular}{|c|c|c|c|c|c|c|}
\hline Gene & SNP & Allele & $\begin{array}{c}\text { PTDM } \\
\mathbf{n}(\%)\end{array}$ & $\begin{array}{c}\text { Non-PTDM } \\
\mathbf{n}(\mathbf{\%})\end{array}$ & OR (95\% CI) & p \\
\hline$L E P$ & rs2167270 & G & $91(84.2 \%)$ & $388(78.5 \%)$ & $0.68(0.39-1.19)$ & 0.182 \\
\hline$\cdot$ & & A & $17(15.8 \%)$ & $106(21.5 \%)$ & & \\
\hline$L E P$ & rs13228377 & A & $91(84.2 \%)$ & $380(76.9 \%)$ & $0.62(0.35-1.08)$ & 0.094 \\
\hline$\cdot$ & & G & $17(15.8 \%)$ & $114(23.1 \%)$ & & \\
\hline$L E P R$ & rs8179183 & G & $97(89.8 \%)$ & $454(91.9 \%)$ & $1.28(0.63-2.59)$ & 0.480 \\
\hline$\cdot$ & & C & $11(10.2 \%)$ & $40(8.1 \%)$ & & \\
\hline$L E P R$ & rs 1137100 & G & $93(86.1 \%)$ & $377(76.3 \%)$ & $1.92(1.02-1.19)$ & 0.025 \\
\hline & & A & $15(13.9 \%)$ & $117(23.7 \%)$ & & \\
\hline$L E P R$ & rs 1137101 & G & $101(94 \%)$ & $421(85 \%)$ & $1.13(1.05-1.23)$ & 0.019 \\
\hline & & A & $7(6 \%)$ & $73(15 \%)$ & & \\
\hline
\end{tabular}

SNPs: single nucleotide polymorphisms; PTDM: post-transplantation diabetes mellitus; LEP: leptin; LEPR: leptin receptor

The $\mathrm{r}^{2}$ values between rs 8179183 and $\mathrm{rs} 1137100$, and between $\mathrm{rs} 8179183$ and rs1137101, between rs1137100 and rs1137101 were 0.328, 0.509, and 0.647 , respectively.

\section{Discussion}

This is the first study to evaluate the genetic association of $L E P R$ and PTDM in renal transplant recipients. Our study demonstrated that two SNPs in LEPR (rs1137100 and rs1137101) were significantly associated with the development of PTDM in Korean renal transplant patients. LEP is a hormone which is synthesized and secreted by adipose tissue, and is known to be important in regulating several neuropeptides and energy homeostasis. It has been reported to play an important role in regulation of body weight, fat metabolism, and glucose uptake $[11,15]$. Patients with T2DM showed decreased LEP expression in adipose tissue, and had lower serum LEP levels [16]. LEP modulates insulin secretion and action via LEPRs present in the hypothalamus, pancreatic cells, adipose tissue, and muscles [17]. In previous studies, LEPR polymorphisms were reported to be associated with diabetes, insulin resistance, metabolic syndrome, and obesity [18-20]. A hypothetical explanation for these results is that polymorphisms of the LEPR result in dysfunction of the LEP-associated signaling pathway and inhibition of the favorable effects of LEP [21].

In addition to the present study, our group previously reported that polymorphisms in AGT, CCL5, IL17E, IL17RA, IL17RB,IL1B, IL2, IL4, IL7R, MMP2, TLR4, and TLR6 were significantly associated with the development of PTDM in Korean renal transplant recipients [10,2225]. Considering the clinical impact of each gene, our previous data suggests that impaired insulin secretion, decreased insulin sensitivity, inflammation of islet $\beta$-cells, and activation of the innate immune system may play essential roles in the pathogenesis of PTDM.

In the last decade, numerous other genetic studies for PTDM have been conducted in renal transplant recipients, and nearly 50 loci have been established as suspected loci (Table 4) [7-10,13,22-41]. Polymorphisms in AIPOQ, CAPN10, CDKAL1, CDKN2A/B, HHEX, KCN11, KCNQ1, SLC30A8, and TCFL2 are known to be associated with T2DM. Yang et al. [32] reported that polymorphisms in IRS1 and HNF4 increased the risk of PTDM in a Hispanic population. Elens et al. [37] showed that PPAR $\alpha$ and POR polymorphisms are significantly associated with PTDM in renal transplant patients treated with tacrolimus. The CYP4F2 gene, which is known to be the main gene involved in creation of 20-hydroxyeicosatetraenoic acid, was also reported as an independent risk factor for PTDM [30]. These genes are associated with decreased insulin secretion through $\beta$-cell impairment 
Table 3. Logistic regression analysis of $L E P$ and $L E P R$ polymorphisms in PTDM and non-PTDM subjects adjusted for age, sex, and tacrolimus usage.

\begin{tabular}{|c|c|c|c|c|c|c|c|}
\hline Gene & SNP & Model & Type & PTDM, n (\%) & Non-PTDM, n (\%) & OR (95\% CI) & $\mathbf{p}$ \\
\hline \multirow{14}{*}{$L E P$} & \multirow{7}{*}{ rs2167270 } & \multirow{3}{*}{ Codominant } & $\mathrm{G} / \mathrm{G}$ & $39(72.2 \%)$ & $147(59.8 \%)$ & \multirow{3}{*}{$0.55(0.27-1.11)$} & \multirow{3}{*}{0.200} \\
\hline & & & $\mathrm{A} / \mathrm{G}$ & $13(24.1 \%)$ & $92(37.4 \%)$ & & \\
\hline & & & $\mathrm{A} / \mathrm{A}$ & $2(3.7 \%)$ & $7(2.8 \%)$ & & \\
\hline & & \multirow{2}{*}{ Dominant } & $\mathrm{G} / \mathrm{G}$ & $39(72.2 \%)$ & $147(59.8 \%)$ & \multirow{2}{*}{$0.60(0.31-1.16)$} & \multirow{2}{*}{0.12} \\
\hline & & & $\mathrm{A} / \mathrm{G}+\mathrm{A} / \mathrm{A}$ & $15(27.8 \%)$ & $99(40.2 \%)$ & & \\
\hline & & \multirow{2}{*}{ Recessive } & $\mathrm{G} / \mathrm{G}+\mathrm{A} / \mathrm{G}$ & $52(96.3 \%)$ & $239(97.2 \%)$ & \multirow{2}{*}{$1.58(0.30-8.24)$} & \multirow{2}{*}{0.60} \\
\hline & & & $\mathrm{A} / \mathrm{A}$ & $2(3.7 \%)$ & $7(2.8 \%)$ & & \\
\hline & \multirow{7}{*}{ rs13228377 } & \multirow{3}{*}{ Codominant } & $\mathrm{A} / \mathrm{A}$ & $39(72.2 \%)$ & $142(57.7 \%)$ & \multirow{3}{*}{$0.54(0.27-1.08)$} & \multirow{3}{*}{0.19} \\
\hline & & & $\mathrm{A} / \mathrm{G}$ & $13(24.1 \%)$ & $94(38.2 \%)$ & & \\
\hline & & & $\mathrm{G} / \mathrm{G}$ & $2(3.7 \%)$ & $10(4.1 \%)$ & & \\
\hline & & \multirow{2}{*}{ Dominant } & $\mathrm{A} / \mathrm{A}$ & $39(72.2 \%)$ & $142(57.7 \%)$ & \multirow{2}{*}{$0.55(0.28-1.07)$} & \multirow{2}{*}{0.073} \\
\hline & & & $\mathrm{A} / \mathrm{G}+\mathrm{G} / \mathrm{G}$ & $15(27.8 \%)$ & $104(42.3 \%)$ & & \\
\hline & & \multirow{2}{*}{ Recessive } & $\mathrm{A} / \mathrm{A}+\mathrm{G} / \mathrm{G}$ & $52(96.3 \%)$ & $236(95.9 \%)$ & \multirow{2}{*}{$0.83(0.17-4.09)$} & \multirow{2}{*}{0.81} \\
\hline & & & $\mathrm{A} / \mathrm{G}$ & $2(3.7 \%)$ & $10(4.1 \%)$ & & \\
\hline \multirow{21}{*}{$L E P R$} & \multirow{7}{*}{ rs8179183 } & \multirow{3}{*}{ Codominant } & $\mathrm{G} / \mathrm{G}$ & $43(79.6 \%)$ & $207(84.2 \%)$ & \multirow{3}{*}{$1.37(0.63-2.97)$} & \multirow{3}{*}{0.55} \\
\hline & & & $\mathrm{C} / \mathrm{G}$ & $11(20.4 \%)$ & $38(15.4 \%)$ & & \\
\hline & & & $\mathrm{C} / \mathrm{C}$ & $0(0 \%)$ & $1(0.4 \%)$ & & \\
\hline & & \multirow{2}{*}{ Dominant } & $\mathrm{G} / \mathrm{G}$ & $43(79.6 \%)$ & $207(84.2 \%)$ & $132(0.61285)$ & 044 \\
\hline & & & $\mathrm{C} / \mathrm{G}+\mathrm{C} / \mathrm{C}$ & $11(20.4 \%)$ & $39(15.8 \%)$ & $1.32(0.01-2.85)$ & 0.44 \\
\hline & & & $\mathrm{G} / \mathrm{G}+\mathrm{C} / \mathrm{G}$ & $54(100 \%)$ & $245(99.6 \%)$ & & \\
\hline & & Recessive & $\mathrm{C} / \mathrm{C}$ & $0(0 \%)$ & $1(0.4 \%)$ & $1.09(0.48-2.47)$ & 0.840 \\
\hline & & & $\mathrm{G} / \mathrm{G}$ & $40(74.1 \%)$ & $145(58.9 \%)$ & & \\
\hline & & Codominant & $\mathrm{A} / \mathrm{G}$ & $13(24.1 \%)$ & $87(35.4 \%)$ & $0.55(0.27-1.11)$ & 0.09 \\
\hline & & & $\mathrm{A} / \mathrm{A}$ & $1(1.8 \%)$ & $14(5.7 \%)$ & & \\
\hline & rs1137100 & Dominant & $\mathrm{G} / \mathrm{G}$ & $40(74.1 \%)$ & $145(58.9 \%)$ & $200(1002-393)$ & 0037 \\
\hline & & Dominant & $\mathrm{A} / \mathrm{G}+\mathrm{A} / \mathrm{A}$ & $14(25.9 \%)$ & $101(41.1 \%)$ & $2.00(1.02-3.93)$ & 0.031 \\
\hline & & Pececine & $\mathrm{G} / \mathrm{G}+\mathrm{A} / \mathrm{G}$ & $53(98.2 \%)$ & $232(94.3 \%)$ & $161(0,80-232)$ & 017 \\
\hline & & Recessive & $\mathrm{A} / \mathrm{A}$ & $1(1.8 \%)$ & $14(5.7 \%)$ & $1.61(0.80-3.23)$ & 0.17 \\
\hline & & & $\mathrm{G} / \mathrm{G}$ & $47(87 \%)$ & $179(72.8 \%)$ & & \\
\hline & & Codominant & $\mathrm{A} / \mathrm{G}$ & $7(13 \%)$ & $62(25.2 \%)$ & $2.48(1.05-5.87)$ & 0.029 \\
\hline & & & $\mathrm{A} / \mathrm{A}$ & $0(0 \%)$ & $5(2 \%)$ & & \\
\hline & rs1137101 & Dominant & $\mathrm{G} / \mathrm{G}$ & $47(87 \%)$ & $179(72.8 \%)$ & $2.69(1.14-6.35)$ & 0.014 \\
\hline & & Dominant & $\mathrm{A} / \mathrm{G}+\mathrm{A} / \mathrm{A}$ & $7(13 \%)$ & $37(27.2 \%)$ & $2.09(1.14-6.35)$ & 0.014 \\
\hline & & & $\mathrm{G} / \mathrm{G}+\mathrm{A} / \mathrm{G}$ & $184(74.8 \%)$ & $47(13 \%)$ & & \\
\hline & & Recessive & $\mathrm{A} / \mathrm{A}$ & $0(0 \%)$ & $5(2 \%)$ & $0.00(0.00-\mathrm{NA})$ & 0.17 \\
\hline
\end{tabular}

PTDM: post-transplantation diabetes mellitus; SNPs: single nucleotide polymorphisms

(CCL2, CCL5, CDKAL1, CDKN2A/B, HNF4A, KCNJ11, KCNQ1, $M M P s, N F A T c 4, S L C 30 A 8$, and TCF7L2), increased peripheral insulin resistance (ADIPOQ, AGT, IRS1, and $L E P$ ), inflammation (ILs, TLR4, and TLR6), and oxidative stress (GPX1). In light of these results, PTDM is caused by an imbalance between insulin secretion and resistance, and $\beta$-cell dysfunction may be a dominant mechanism.

However, most of these studies are underpowered and were conducted in relatively small populations. To overcome these limitations, alternative approaches such as genome-wide association studies (GWAS) and meta-analyses are performed. McCaughan et al. [2] performed GWAS with secondary validation. They reported
26 SNPs that were associated with PTDM, and the association was validated for 8 SNPs. These SNPs were associated with apoptosis of beta cells, and the authors suggested that beta cell dysfunction and death play a crucial role in the pathogenesis of PTDM. Benson et al. [6] conducted a comprehensive meta-analysis of 18 polymorphisms in 12 genes which were reported to be genetic markers of PTDM. Of these various polymorphisms, CDKAL1 rs10946398, KCNQ1 rs2237892, and TCF7L2 rs7903146 were significantly associated with PTDM $(\mathrm{p}<0.05)$.

In conclusion, we demonstrated a significant association between LEPR polymorphisms and the development of PTDM in Korean renal transplant patients. Considering our present results and the above 
Table 4. Previous candidate gene studies evaluating genetic susceptibility to PTDM in renal transplant recipients.

\begin{tabular}{|c|c|c|c|c|c|}
\hline Gene & SNPs & Ethnicity & PTDM Case & Control & References \\
\hline \multirow[t]{2}{*}{ ADIPOQ } & rs1501299 & Asian (Korean) & 154 & 421 & Kang et al. [7] \\
\hline & rs1501299 & Caucasian & 83 & 187 & Nicoletto et al. [26] \\
\hline$A G T$ & rs4762 & Asian (Korean) & 49 & 253 & Lee et al. [22] \\
\hline CAPN10 & rs5030952 & Caucasian & 56 & 158 & Kurzawski et al. [27] \\
\hline CCL2 & rs1024611 & Caucasian & 43 & 272 & Dabrowska-Zamojcin et al. [28] \\
\hline \multirow[t]{3}{*}{ CCL5 } & rs2107538 & Asian (Korean) & 56 & 255 & Jeong et al. $[10]$ \\
\hline & rs2280789 & Asian (Korean) & 56 & 255 & Jeong et al. [10] \\
\hline & rs3817655 & Asian (Korean) & 56 & 255 & Jeong et al. [10] \\
\hline CDKAL1 & rs 10946398 & Asian (Korean) & 145 & 444 & Kang et al. [29] \\
\hline$C D K N 2 A / B$ & rs10811661 & Asian (Korean) & 145 & 444 & Kang et al. [29] \\
\hline CYP4F2 & rs 2108622 & Caucasian & 34 & 130 & Gervasini et al. [30] \\
\hline GPX1 & rs1050450 & Caucasian & 21 & 138 & Dutkiewicz et al. [31] \\
\hline \multirow[t]{3}{*}{ HHEX } & rs1111875 & Asian (Korean) & 145 & 444 & Kang et al. [29] \\
\hline & rs 5015480 & Asian (Korean) & 145 & 444 & Kang et al. [29] \\
\hline & rs7923837 & Asian (Korean) & 145 & 444 & Kang et al. [29] \\
\hline \multirow[t]{2}{*}{ HNF4A } & rs 1884614 & Hispanic & 133 & 170 & Yang et al. [32] \\
\hline & rs2144908 & Hispanic & 133 & 170 & Yang et al. [32] \\
\hline$I L 17 E$ & rs1124053 & Asian (Korean) & 53 & 253 & Kim et al. [23] \\
\hline$I L 17 F$ & rs763780 & Caucasian & 23 & 146 & Romanowski et al. [33] \\
\hline \multirow[t]{2}{*}{ IL17RA } & rs2229151 & Asian (Korean) & 53 & 253 & Kim et al. [23] \\
\hline & rs4819554 & Asian (Korean) & 53 & 253 & Kim et al. [23] \\
\hline \multirow[t]{2}{*}{$I L 17 R B$} & rs 1025689 & Asian (Korean) & 53 & 253 & Kim et al. [23] \\
\hline & rs1043261 & Asian (Korean) & 53 & 253 & Kim et al. [23] \\
\hline$I L 1 B$ & rs3136558 & Asian (Korean) & 53 & 253 & Kim et al. [23] \\
\hline \multirow[t]{2}{*}{$I L 2$} & rs2069762 & Asian (Korean) & 53 & 253 & Kim et al. [23] \\
\hline & rs2069763 & Asian (Korean) & 53 & 253 & Kim et al. [23] \\
\hline \multirow[t]{2}{*}{ IL4 } & rs2070874 & Asian (Korean) & 53 & 253 & Kim et al. [23] \\
\hline & rs 2243250 & Asian (Korean) & 53 & 253 & Kim et al. [23] \\
\hline IL6 & rs1800795 & Caucasian & 59 & 302 & Bamoulid et al. [41] \\
\hline \multirow[t]{2}{*}{$I L 7 R$} & rs1494558 & Asian (Korean) & 53 & 253 & Kim et al. [23] \\
\hline & rs2172749 & Asian (Korean) & 53 & 253 & Kim et al. [22] \\
\hline IRS1 & rs1801278 & Hispanic & 133 & 170 & Yang et al. [32] \\
\hline \multirow[t]{2}{*}{ KCNJI1 } & rs5219 & Caucasian & 115 & 205 & Tavira et al. [34] \\
\hline & rs5210 & Asian (Indian) & 140 & 500 & Khan et al. [9] \\
\hline \multirow[t]{3}{*}{ KCNQ1 } & rs 2237892 & Asian (Korean) & 145 & 444 & Kang et al. [29] \\
\hline & rs2237895 & Caucasian & 145 & 260 & Tavira et al. [35] \\
\hline & rs2283228 & Asian (Indian) & 140 & 500 & Khan et al. [9] \\
\hline$L E P$ & re2167270 & Caucasian & 43 & 280 & Romanowski et al. [30] \\
\hline \multirow[t]{2}{*}{ MMP2 } & rs1132896 & Asian (Korean) & 52 & 257 & Ong et al. [24] \\
\hline & rs243849 & Asian (Korean) & 52 & 257 & Ong et al. [24] \\
\hline NFATc4 & rs 10141896 & Hispanic & 162 & 157 & Chen et al. [36] \\
\hline POR & rs 1057868 & Caucasian & 9 & 76 & Elens et al. [37] \\
\hline PPAR $\alpha$ & rs4253728 & Caucasian & 9 & 76 & Elens et al. [37] \\
\hline \multirow[t]{2}{*}{ SLC30A8 } & rs 13266634 & Asian (Korean) & 174 & 450 & Kang et al. [38] \\
\hline & rs 13266634 & Asian (Indian) & 42 & 98 & Khan et al. [39] \\
\hline \multirow[t]{3}{*}{ TCF $7 L 2$} & rs7903146 & Asian (Korean) & 119 & 392 & Kang et al. [40] \\
\hline & rs7903146 & Caucasian & 114 & 958 & Ghisdal et al. [8] \\
\hline & rs7903146 & Asian (Indian) & 42 & 98 & Khan et al. [39] \\
\hline TLR4 & rs1927914 & Asian (Korean) & 51 & 254 & Kim et al. [25] \\
\hline TLR6 & rs1039559 & Asian (Korean) & 51 & 254 & Kim et al. [25] \\
\hline
\end{tabular}

PTDM: post-transplantation diabetes mellitus; SNPs: single nucleotide polymorphisms; ADIPOQ: adiponectin; AGT: angiotensinogen; CAPN10: calpain-10 gene; CCL2: C-C motif chemokine ligand 2; CCL5: C-C motif chemokine ligand 5; CDKAL1 cyclin-dependent kinase 5 regulatory subunit-associated protein 1-like 1; CDKN2A/B: cyclin-dependent kinase inhibitor-2A/B; CYP4F2: cytochrome P450 family 4 subfamily F member 2; GPX1: glutathione peroxidase 1; HHEX: hematopoietically expressed homeobox; HNF4A: hepatocyte nuclear factor 4 alpha; IL: interleukin; IRS1: insulin receptor substrate 1; KCNJ11: potassium voltage-gated channel subfamily J member 11; KCNQ1: potassium voltage-gated channel subfamily Q member 1; LEP: leptin; MMP2: matrix metalloproteinase 2; NFATc4: nuclear factor of activated T cells: cytoplasmic: calcineurin dependent 4; POR. P450 oxidoreductase; PPAR $\alpha$, $\mathrm{p}$ eroxisome proliferator-activated receptor $\alpha$; SLC30A8: solute carrier member 3 zinc transporter member 8; TCF7L2: transcription factor 7 like 2; TLR; toll-like receptor 
mentioned studies, genetic susceptibility plays an essential role in the pathogenesis of PTDM. Discovery of precise genetic polymorphisms that impact the development of PTDM is important to understanding its mechanisms and contributing to early diagnosis and proper management of the condition. Prospective, large-scale investigations that include assessment of the biological effects of gene polymorphisms are needed.

\section{References}

1. Ghisdal L, Van Laecke S, Abramowicz MJ, Vanholder R, Abramowicz D (2012) Newonset diabetes after renal transplantation: risk assessment and management. Diabetes Care 35: 181-188. [Crossref]

2. McCaughan JA, McKnight AJ, Maxwell AP (2014) Genetics of new-onset diabetes after transplantation. J Am Soc Nephrol 25: 1037-1049. [Crossref]

3. Kesiraju S, Paritala P, Rao Ch UM, Sahariah S (2014) New onset of diabetes after transplantation - an overview of epidemiology, mechanism of development and diagnosis. Transpl Immunol 30(1): 52-58.

4. van Hooff JP, Christiaans MH, van Duijnhoven EM (2004) Evaluating mechanisms of post-transplant diabetes mellitus. Nephrol Dial Transplant 19 Suppl 6: vi8-8vi12. [Crossref]

5. Palepu S, Prasad GV (2015) New-onset diabetes mellitus after kidney transplantation: Current status and future directions. World J Diabetes 6: 445-455.

6. Benson KA, Maxwell AP, McKnight AJ (2016) A HuGE Review and Meta-Analyses of Genetic Associations in New Onset Diabetes after Kidney Transplantation. PLoS One 11: e0147323. [Crossref]

7. Kang ES, Magkos F, Kim BS, Zhai R, Su L, et al. (2012) Variants of the adiponectin and adiponectin receptor-1 genes and posttransplantation diabetes mellitus in renal allograft recipients. J Clin Endocrinol Metab 97: E129-135.

8. Ghisdal L, Baron C, Le Meur Y, Lionet A, Halimi JM, et al. (2009) TCF7L2 polymorphism associates with new-onset diabetes after transplantation. $J$ Am Soc Nephrol 20: 2459-2467. [Crossref]

9. Imran Ali Khan KKV, Parveen Jahan, Kamal Kiran Mukkavali, Qurratulain Hasan, Pragna Rao (2015) Correlation between KCNQ1 and KCNJ11 gene polymorphisms and type 2 and post-transplant diabetes mellitus in the Asian Indian population. Genes \& Diseases 2: 276-282.

10. Jeong KH, Moon JY, Chung JH, Kim YH, Lee TW (2010) Significant associations between CCL5 gene polymorphisms and post-transplantational diabetes mellitus in Korean renal allograft recipients. Am J Nephrol 32: 356-361.

11. Wauters M, Mertens I, Rankinen T, Chagnon M, Bouchard C, et al. (2001) Leptin receptor gene polymorphisms are associated with insulin in obese women with impaired glucose tolerance. J Clin Endocrinol Metab 86: 3227-3232. [Crossref]

12. de Luis DA, Gonzalez Sagrado M, Aller R, Izaola O, Conde R (2008) Influence of Lys656Asn polymorphism of leptin receptor gene on insulin resistance in patients with diabetes mellitus type 2. Diabetes Res Clin Pract 81: e9-e11.

13. Romanowski M, Dziedziejko V, Maciejewska-Karlowska A, Sawczuk M, Safranow $\mathrm{K}$, et al. (2015) Adiponectin and leptin gene polymorphisms in patients with posttransplant diabetes mellitus. Pharmacogenomics 16: 1243-1251.

14. Genuth S, Alberti KG, Bennett P, Buse J, Defronzo R, et al. (2003) Follow-up report on the diagnosis of diabetes mellitus. Diabetes Care 26: 3160-3167. [Crossref]

15. Wauters M, Considine RV, Van Gaal LF (2000) Human leptin: from an adipocyte hormone to an endocrine mediator. Eur J Endocrinol 143: 293-311. [Crossref]

16. Dardeno TA, Chou SH, Moon HS, Chamberland JP, Fiorenza CG, et al. (2010) Leptin in human physiology and therapeutics. Front Neuroendocrinol 31: 377-393. [Crossref]

17. Seufert J, Kieffer TJ, Leech CA, Holz GG, Moritz W, et al. (1999) Leptin suppression of insulin secretion and gene expression in human pancreatic islets: implications for the development of adipogenic diabetes mellitus. J Clin Endocrinol Metab 84: 670-676.

18. Su S, Zhang C, Zhang F, Li H, Yang X, et al. (2016) The association between leptin receptor gene polymorphisms and type 2 diabetes mellitus: A systematic review and meta-analysis. Diabetes Res Clin Pract 121: 49-58.

19. Gottlieb MG, Bodanese LC, Leite LE, Schwanke CH, Piccoli Jda C, et al. (2009) Association between the Gln223Arg polymorphism of the leptin receptor and metabolic syndrome in free-living community elderly. Metab Syndr Relat Disord 7: 341-348.
20. Furusawa T, Naka I, Yamauchi T, Natsuhara K, Kimura R, et al. (2010) The Q223R polymorphism in LEPR is associated with obesity in Pacific Islanders. Hum Genet 127: 287-294. [Crossref]

21. Lakka TA, Rankinen T, Weisnagel SJ, Chagnon YC, Lakka HM, et al. (2004) Leptin and leptin receptor gene polymorphisms and changes in glucose homeostasis in response to regular exercise in nondiabetic individuals: the HERITAGE family study. Diabetes 53: 1603-1608.

22. Lee, Sr., Moon JY, Lee SH, Ihm CG, Lee TW, et al. (2013) Angiotensinogen polymorphisms and post-transplantation diabetes mellitus in Korean renal transplan subjects. Kidney Blood Press Res 37: 95-102.

23. Kim YG, Ihm CG, Lee TW, Lee SH, Jeong KH, et al. (2012) Association of genetic polymorphisms of interleukins with new-onset diabetes after transplantation in renal transplantation. Transplantation 93: 900-907.

24. Ong S, Kang SW, Kim YH, Kim TH, Jeong KH, et al. (2016) Matrix Metalloproteinase Gene Polymorphisms and New-Onset Diabetes After Kidney Transplantation in Korean Renal Transplant Subjects. Transplant Proc 48: 858-863.

25. Kim JS, Kim SK, Park JY, Kim YG, Moon JY, et al. (2016) Significant Association between Toll-Like Receptor Gene Polymorphisms and Posttransplantation Diabetes Mellitus. Nephron 133: 279-286.

26. Nicoletto BB, Souza GC, Fonseca NK, Centenaro A, Manfro RC, et al. (2013) Association between $276 \mathrm{G} / \mathrm{T}$ adiponectin gene polymorphism and new-onset diabetes after kidney transplantation. Transplantation 96: 1059-1064.

27. Kurzawski M, Dziewanowski K, Kedzierska K, Gornik W, Banas A, et al. (2010) Association of calpain-10 gene polymorphism and posttransplant diabetes mellitus in kidney transplant patients medicated with tacrolimus. Pharmacogenomics $J$ 10: 120-125.

28. Dabrowska-Zamojcin E, Romanowski M, Dziedziejko V, Maciejewska-Karlowska A, Sawczuk M, et al. (2016) CCL2 gene polymorphism is associated with post-transplant diabetes mellitus. Int Immunopharmacol 32: 62-65. [Crossref]

29. Kang ES, Kim MS, Kim CH, Nam CM, Han SJ, et al. (2009) Association of common type 2 diabetes risk gene variants and posttransplantation diabetes mellitus in renal allograft recipients in Korea. Transplantation 88: 693-698.

30. Gervasini G, Luna E, Garcia-Cerrada M, Garcia-Pino G, Cubero JJ (2016) Risk factors for post-transplant diabetes mellitus in renal transplant: Role of genetic variability in the CYP450-mediated arachidonic acid metabolism. Mol Cell Endocrinol 419: 158-164.

31. Dutkiewicz G, Domanski L, Pawlik A, Binczak-Kuleta A, Safranow K, et al. (2010) Polymorphisms of superoxide dismutase, glutathione peroxidase and catalase genes in patients with post-transplant diabetes mellitus. Arch Med Res 41: 350-355.

32. Yang J, Hutchinson, II, Shah T, Min DI (2011) Genetic and clinical risk factors of new-onset diabetes after transplantation in Hispanic kidney transplant recipients. Transplantation 91: 1114-1119.

33. Romanowski M, Domanski L, Pawlik A, Osekowska B, Dziedziejko V, et al. (2015) Interleukin-17 gene polymorphisms in patients with post-transplant diabetes mellitus. Eur Rev Med Pharmacol Sci 19: 3152-3156. [Crossref]

34. Tavira B, Coto E, Torres A, Diaz-Corte C, Diaz-Molina B, et al. (2012) Association between a common KCNJ11 polymorphism (rs5219) and new-onset posttransplant diabetes in patients treated with Tacrolimus. Mol Genet Metab 105: 525-527.

35. Tavira B, Coto E, Díaz-Corte C, Ortega F, Arias M, et al. (2011) KCNQ1 gene variants and risk of new-onset diabetes in tacrolimus-treated renal-transplanted patients. Clin Transplant 25: E284-291. [Crossref]

36. Chen Y, Sampaio MS, Yang JW, Min D, Hutchinson IV (2012) Genetic polymorphisms of the transcription factor NFATc4 and development of new-onset diabetes after transplantation in Hispanic kidney transplant recipients. Transplantation 93: 325-330.

37. Elens L, Sombogaard F, Hesselink DA, van Schaik RH, van Gelder T (2013) Singlenucleotide polymorphisms in $\mathrm{P} 450$ oxidoreductase and peroxisome proliferatoractivated receptor-alpha are associated with the development of new-onset diabetes after transplantation in kidney transplant recipients treated with tacrolimus. Pharmacogenet Genomics 23: 649-657.

38. Kang ES, Kim MS, Kim YS, Kim CH, Han SJ, et al. (2008) A polymorphism in the zinc transporter gene SLC30A8 confers resistance against posttransplantation diabetes mellitus in renal allograft recipients. Diabetes 57: 1043-1047.

39. Khan IA, Jahan P, Hasan Q, Rao P (2015) Validation of the association of TCF7L2 and SLC30A8 gene polymorphisms with post-transplant diabetes mellitus in Asian Indian population. Intractable Rare Dis Res 4: 87-92. 
Kim JS (2017) Association of leptin receptor gene polymorphisms with post-transplant diabetes mellitus: Short report and literature review

40. Kang ES, Kim MS, Kim YS, Hur KY, Han SJ, et al. (2008) A variant of the transcription factor 7-like 2 (TCF7L2) gene and the risk of posttransplantation diabetes mellitus in renal allograft recipients. Diabetes Care 31: 63-68.
41. Bamoulid J, Courivaud C, Deschamps M, Mercier P, Ferrand C, et al. (2006) IL-6 promoter polymorphism -174 is associated with new-onset diabetes after transplantation. J Am Soc Nephrol 17: 2333-2340. [Crossref]

Copyright: $(02017$ Kim JS. This is an open-access article distributed under the terms of the Creative Commons Attribution License, which permits unrestricted use, distribution, and reproduction in any medium, provided the original author and source are credited. 